\title{
DESIGN OF ANTIPROTON ELECTRON COOLING IN THE RECYCLER
}

\author{
A. Burov, J. MacLachlan, J. Marriner and S. Nagaitsev, FNAL, Batavia, IL
}

\section{Abstract}

A conceptual design of electron cooling of $9 \mathrm{GeV}$ antiprotons for the Tevatron is discussed. Analytic and numeric calculations of the cooling process determine the basic requirements of the cooler.

\section{PURPOSE}

During a Tevatron store, emittances of the colliding bunches grow and the luminosity decreases. For RUN II, the luminosity is calculated to drop by a factor of 2 after 6-7 hours [1], so the beams should be renewed after this time. The purpose of the Recycler storage ring is to accept the unspent antiprotons $(\bar{p})$ from the Tevatron, to recool them transversely and longitudinally, and to redeliver them to the collider. The Recycler must also accept fresh antiprotons from the Accumulator, and this process requires longitudinal cooling too. For RUN II, the stochastic cooling system is thought to be adequate; an example of simulations for the transverse cooling is shown in Fig. 1.

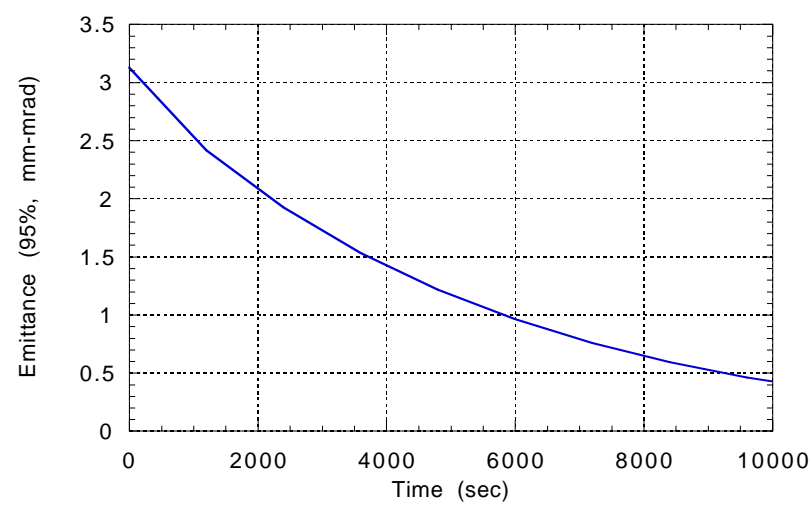

Figure 1: Evolution of the (unnormalized) emittance of $5 \cdot 10^{12}$ particles in the Recycler with the full momentum spread $\pm 2 \cdot 10^{-3}$ during stochastic cooling with $2-4 \mathrm{GHz}$ bandwidth.

However, the efficiency of the stochastic cooling decreases with intensity of the cooled beam, which could make it insufficient for the future $\mathrm{TeV} 33$ program. Electron cooling might be a good supplement to stochastic cooling because of its independence on the beam intensity. On the other hand, electron cooling is much more efficient if the beam is already precooled. Thus, a hybrid cooling scheme could be used in the Recycler, with stochastic cooling for the first stage and electron cooling for the second.

\section{SCENARIO}

A possible scenario for the periodic cooling-stacking process in the Recycler could be following:

- $t=0: 100$ bunches of (hot) antiprotons leave the Tevatron, are decelerated in the Main Injector, and arrive at the Recycler, sharing its circumference with already cooled antiproton beam. Then, the cold portion is transfered to the Main Injector, releasing the phase space for the hot beam with $N=(2.5-10) \cdot 10^{12} \vec{p}$ s occupying $\mathcal{A}=400 \mathrm{eVs}$ of the longitudinal phase space and $30 \pi \mathrm{mm}$ mrad of the normalized $95 \%$ emittance. Transverse stochastic precooling starts.

- Every quarter of an hour, a fresh pbar batch arrives from the Accumulator. Its population is $10^{11}$ in $10 \mathrm{eVs}$ and $15 \pi \mathrm{mm}$ mrad (normalized 95\%). It is adjoined longitudinally to the whole stack by means of the barrier-bucket technique [2].

- $t=1-2 \mathrm{~h}$ : Stochastic precooling finishes; beam emittance is $15 \pi \mathrm{mm}$ mrad. Electron cooling begins.

- $t=3-8 \mathrm{~h}$ : Electron cooling finishes producing a beam with $10 \pi$ emittance and $150 \mathrm{eVs}$ or less of the longitudinal phase space. The cycle is then repeated.

\section{SIMULATION RESULTS}

To simulate electron cooling processes, a multi-particle $\mathrm{C}++$ code has been written. This code tracks the time evolution of an ensemble of cooled particles, optimizes the cooling process under various conditions and finds the tolerances on imperfections.

In distinction to usual situation in low-energy coolers, relative velocities between the cooled particles and the electrons are supposed here to be determined by the cooled particle $(\bar{p})$ velocities. Electron velocities are assumed to be low enough not to depress the cooling rates. In this case, the longitudinal and transverse cooling rates $\lambda_{\|}$and $\lambda_{\perp}$ are strong functions of the $\bar{p}$ longitudinal and transverse velocities in the beam frame $v_{\|}$and $v_{\perp}$ [3]:

$$
\lambda_{\|} \propto\left(v_{\perp}^{2} v_{\|}\right)^{-1}, \quad \lambda_{\perp} \propto v_{\perp}^{-3} \quad \text { for } v_{\|} \leq v_{\perp} .
$$

The smaller the $\bar{p}$ velocity is, the faster it decreases. Thus, electron cooling tends to shape a narrow core of supercooled particles inside the distributions. For flat pbar distributions, i.e., $v_{\|} \ll v_{\perp}$, the longitudinal rates are higher than the transverse and the longitudinal core is created first. Actual size of this core is determined by an equilibrium between cooling and IBS diffusion which is calculated separately (see section 5). The evolution of the recycled $\vec{p}$ s from the initial state is shown in Figs. 2 and 3. 


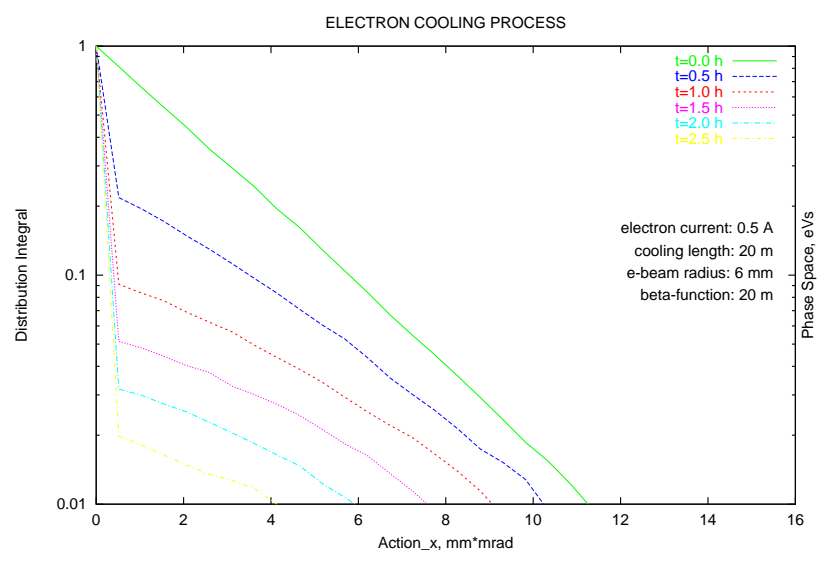

Figure 2: Evolution of the transverse $\bar{p}$ distribution from the initial Gaussian one with the rms normalized emittance $2.5 \mathrm{~mm}$ mrad.

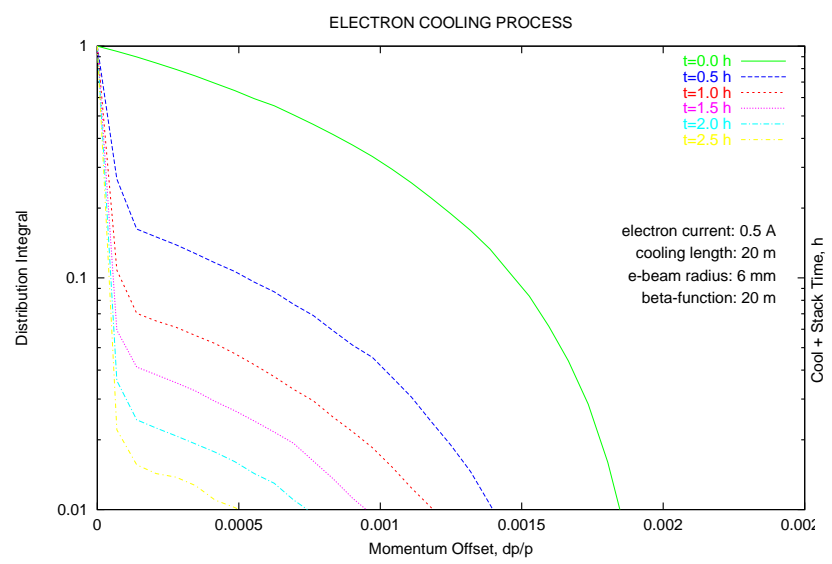

Figure 3: Evolution of the $\bar{p}$ momentum distribution from an initial parabolic one.

The conclusion from the simulations is that transverse cooling of the recycled $\bar{p}$ 's from $\epsilon=15 \pi \mathrm{mm}$ mrad to $\epsilon=10 \pi \mathrm{mm}$ mrad requires 0.9 Ampere $\times$ hour (Ah) of (cooler length $) \times($ cooling time $)$, for a $20 \mathrm{~m}$ cooling section. For beam from the Accumulator, this value is $0.7 \mathrm{Ah}$.

The longitudinal phase area $\mathcal{A}$ shrinks with a rate $r_{0} \approx$ $1.2 \mathrm{~A}^{-1} \mathrm{~h}^{-1}$ over the whole interesting interval $150 \mathrm{eVs}<$ $\mathcal{A}<400 \mathrm{eVs}$. This approximate rate is used in the stacking model discussed below.

\section{COOLING-STACKING PROCESS}

The phase space evolution in the cooling-stacking process can be described as:

$$
\dot{\mathcal{A}}=-r_{0} \mathcal{A}+f_{s} \mathcal{A}_{b}+f_{s} \Delta_{s} \mathcal{A}
$$

where $f_{s}$ is the stacking frequency, the number of injections per hour, $\mathcal{A}_{b}$ is the batch phase area, and $\Delta_{s}$ is the fractional

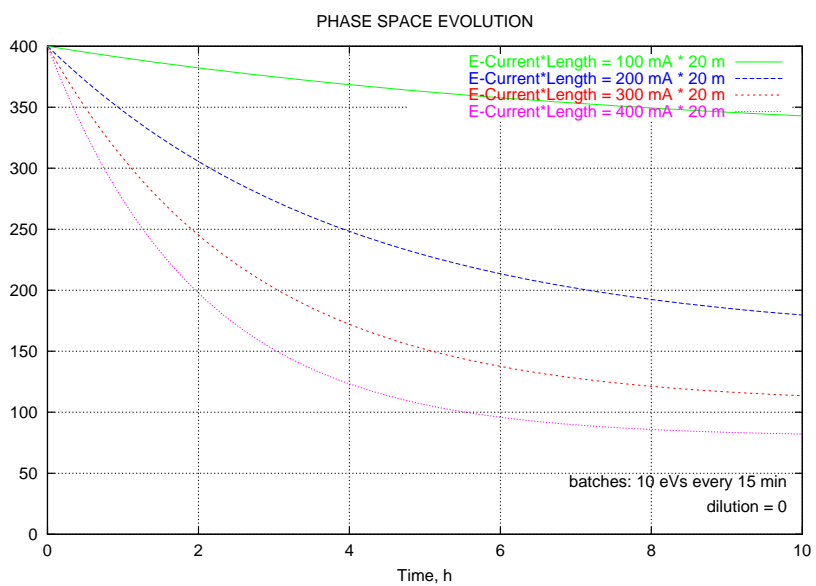

Figure 4: Evolution of the longitudinal phase space area in the cooling-stacking process

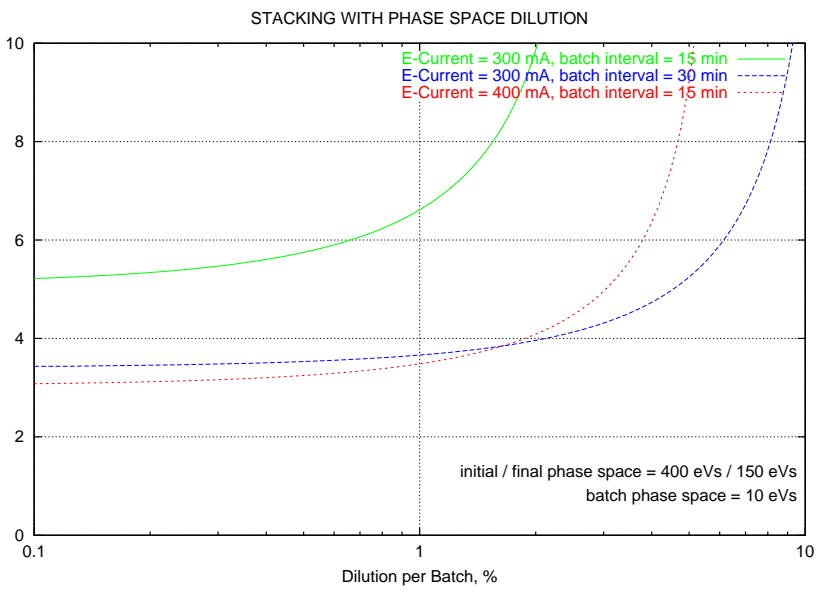

Figure 5: Tolerances on the longitudinal phase space dilution due to the stacking imperfection.

phase area dilution due to the stacking imperfection. The solution reads:

$$
\begin{aligned}
& \mathcal{A}(t)=\mathcal{A}(\infty)+(\mathcal{A}(0)-\mathcal{A}(\infty)) \exp \left(-\left(r_{0}-f_{s} \Delta_{s}\right) t\right) \\
& \mathcal{A}(t) \rightarrow \mathcal{A}(\infty)=\mathcal{A}_{b} f_{s} /\left(r_{0}-f_{s} \Delta_{s}\right)
\end{aligned}
$$

The asymptotic phase space area $\mathcal{A}(\infty)$ is related to an equilibrium between factors which tend to increase the phase space (stacking, dilution) and the factor tending to shrink it (cooling). The phase area evolution is presented in Fig. 4, a tolerance for the dilution can be found from the results shown in Fig. 5. The conclusion is that for the injection frequency $f_{s}=4 h^{-1}$ and dilution $\Delta_{s}<1 \%$, the current $I_{e}=300 \mathrm{~mA}$ is sufficient for antiproton accumulation. 


\section{INTRA-BEAM SCATTERING OF ANTIPROTONS}

Intra-beam scattering (IBS) calculations are simplified by assuming that first, the longitudinal $\bar{p}$ velocities are smaller than transverse (typical for hadron beams), and second, beam envelope variations are small enough (about $30 \%$ in the Recycler) to apply the smooth approximation.

Under these assumptions, IBS is reduced to a heat transfer from the hot transverse degrees of freedom to the cold longitudinal one which can be described as a diffusion in the longitudinal degree of freedom. The diffusion imposes a limit on the width of the longitudinal distribution: it cannot be smaller than the one determined by the cooling-diffusion equilibrium. If the equilibrium width is smaller than the design value, IBS can be neglected; otherwise, IBS puts a limit on the final width of the distribution.

Conventionally, the IBS diffusion coefficient is calculated as a single number for the whole distribution. This number results from an averaging of the local diffusion coefficient, which depends on actions of the scattered particle, over the distribution. However, to derive the equilibrium distribution with a good accuracy, a knowledge of the average diffusion is not sufficient; more detailed information contained in the local diffusion dependencies is required. The diffusion coefficient $D$ as a function of velocity amplitudes $v_{x}, v_{y}$ of the given particle which scatters on other particles distributed with rms velocity $v_{\perp}$ is calculated from the Landau collision integral [3]. For a Gaussian transverse distribution

$$
\begin{aligned}
& D=\frac{4 \pi n_{\bar{p}} r_{p}^{2} L_{\bar{p}}}{\gamma^{2} \beta^{2} v_{\perp}} \mathcal{I}, \quad v_{\perp}=\sqrt{\frac{\gamma \epsilon_{n}}{\beta_{f}}} \\
& \mathcal{I}=\frac{2}{\pi} \frac{v_{\perp}^{3}}{\sqrt{\left(v_{x}^{2}+v_{y}^{2}+2 v_{\perp}^{2} / \pi\right)\left(v_{x}^{2}+2 v_{\perp}^{2} / \pi\right)\left(v_{y}^{2}+2 v_{\perp}^{2} / \pi\right)}}
\end{aligned}
$$

where $n_{\bar{p}}$ is the pbar density at the beam axis, $L_{\bar{p}}$ is the IBS Coulomb logarithm and $\epsilon_{n}$ is the normalized rms emittance. The calculated diffusion is consistent with the average diffusion coefficient reported in [4] to an accuracy better than $10 \%$; it is also consistent with numerical calculations of the average diffusion that are free from the above simplifications [5].

The evolution of the longitudinal distribution is described by the Fokker-Planck equation:

$$
\frac{\partial f}{\partial t}=\frac{\partial}{\partial w}\left(F f+\frac{D}{2} \frac{\partial f}{\partial w}\right) ; \quad w=\delta p / p
$$

Here

$$
F=\frac{8\left(I_{e} / e\right) r_{e} r_{p} \eta L_{\|}}{\pi \beta^{2} \gamma^{2} a^{2} v_{x} v_{y}}
$$

is the cooling force independent in this case of the value of the longitudinal velocity $w$, with $I_{e}$ as the electron current, $a$ as the electron beam radius, $\eta$ as the circumference fraction occupied by the cooler and $L_{\|}$as the Coulomb logarithm for the longitudinal cooling [3].
The Fokker-Planck equation (3) with the diffusion coefficient (2) and the cooling force (4) can be analytically resolved for the equilibrium distribution:

$$
f=\exp (-w / \bar{w}) / \bar{w}, \quad \bar{w}=D / 2 F,
$$

yielding the equilibrium longitudinal phase space (95\%) averaged over the transverse distribution

$$
\mathcal{A}=\frac{1}{4} \frac{I_{\bar{p}}}{\eta I_{e}} \frac{r_{p}}{r_{e}} \frac{L_{\bar{p}}}{L_{\|}} \frac{a^{2}}{a_{\bar{p}}^{2}} E T_{0} .
$$

This phase space is sufficiently small; for $N_{\bar{p}}=1 \cdot 10^{13}$ and $I_{e}=300 \mathrm{~mA}, \mathcal{A}=30 \mathrm{eVs}$. As long as the desired phase space area is larger than this equilibrium, the IBS may be neglected.

The main parameters of the electron cooling in the Recycler for the 100 bunch scenario are summarized in Tab. 1.

Table 1: Electron Cooling for 100 Bunches

\begin{tabular}{|l|c|}
\hline Parameter & Value \\
\hline \hline Circumference & $3319.4 \mathrm{~m}$ \\
\hline Pbar momentum & $8.9 \mathrm{Gev} / \mathrm{c}$ \\
\hline Number of pbars, total & $(5-10) \cdot 10^{12}$ \\
\hline Init. long. area recycled pbars & $400 \mathrm{eVs}$ \\
\hline Fin. long. 98\% area, goal & $150 \mathrm{eVs}$ \\
\hline Init. norm. 95\% emittance & $30 \pi \mathrm{mm} \mathrm{mrad}$ \\
\hline Fin. norm. 95\% emittance & $10 \pi \mathrm{mm} \mathrm{mrad}$ \\
\hline Batches per hour & $4 h^{-1}$ \\
\hline Batch area & $10 \mathrm{eVs}$ \\
\hline Batch norm. 95\% emittance & $15 \pi \mathrm{mm} \mathrm{mrad}$ \\
\hline Cooling length & $20 \mathrm{~m}$ \\
\hline Beta-function in the cooler & $20 \mathrm{~m}$ \\
\hline Electron current & $300 \mathrm{~mA}$ \\
\hline Time of cool-stack cycle & $7 \mathrm{hour}$ \\
\hline Electron beam radius & $0.6 \mathrm{~cm}$ \\
\hline Electron angle & $\leq 80 \mu \mathrm{rad}$ \\
\hline Electron temperature & $\leq 1 \mathrm{eV}$ \\
\hline Electron momentum spread & $\leq 5 \cdot 10^{-5}$ \\
\hline
\end{tabular}

The authors are thankful to Ya. S. Derbenev and V. V. Parkhomchuk for fruitful discussions.

\section{REFERENCES}

[1] G. Jackson, ed. "Fermilab Recycler Ring, TDR", Apr. 1996.

[2] J. Griffin et al., IEEE Trans Nucl. Sci. NS-30, p. 3502 (1983).

[3] A. Burov, J. MacLachlan, "Optimization of Electron Cooling for a Medium Energy Accumulator Ring", submitted to NIM A.

[4] V. Lebedev et al., "Single and Multiple Intrabeam Scattering in a Laser Cooled Beam" NIM A 391, p. 176 (1997).

[5] P. Colestock, private communication. 